\title{
Research on Detection and Tracking of Player in Broadcast Sports Video
}

\author{
Yang Wang ${ }^{1}$, Yueqiu Han $^{2}$ and Deming Zhang ${ }^{3}$ \\ ${ }^{1}$ Physical Education Department, Harbin Engineering University, Harbin 150000, \\ China \\ ${ }^{2}$ College of International Cooperation Education, Harbin Engineering University, \\ Harbin 150000, China \\ ${ }^{3}$ Heilongjiang University of Chinese Medicine,Harbin 150000, China \\ Wangyangwy2014@126.com
}

\begin{abstract}
The paper presents a method which bases on support vector machine(SVM) and particle filtering for detecting and tracking player in the broadcalst sports video. Firstly, through the combination of Support Vector Classification and Courtsegmentationmethod, it proposes the algorithm for examining automatically members in those videos, which is used to initialize the trace of subsequent visual objects; secondly, by combing support vector regression frame and the one of sequential Monte Carlo, it bring forth the improved particle filtering algorithm which is applied to follow visual objects, enabling the taditional particle filtering method to achieve robust trail of such targets even when the particle set is small, together with effective enhancement of the running efficien y of tracking system.
\end{abstract}

Keywords: Object tracking; Support Vector Regression; Support vector Classification; Particle Filter; Sportsvideo

\section{Introduction}

The semantic understanding of sports video can essentially be considered to comprehend the progress of sports events [1-2]. As main participants and actors in the event, player behaviors and performance like their footwork and actions in the competition matter a lot to the understanding of the gane progress. As an important behavioral information in the event, player location at one time point on the playground or running trail in some time range can provide important semantic clues to the understanding, event detection, extraction of wonderful clips, content enhancement and tactical analysis of sports video[3-4]. So as the instrument to obtain information about player position and trajectory, the detection and tracking technique used in the sports video has been increasingly concerned by more and more scholars[5-6].

Inspection and tracking of motional objects is key topic in the visual field. The core idea is to utilize integrative techniques for image processing, video analysis etc., to quickly and accurately capture objects in motion. So far, most of existing techniques are mainly used in target monitoring field [7-8]. In that application, targets are moving at low speed. Backgrounds are simple. And in most cases, several static cameras are used to take videos of targets. But, the application environment where object detection and track is made in the airing sports video differs a lot from the video surveillance, on account of the following reasons. Firstly, broadcast sports video are composed by professional compilers after selection and conversion of shooting signals by many non-static cameras. It's inevitable that 
different shot cuts exist in the video flow. Secondly, in the videos, moving objects (player) are moving fast and they appear in different places in the cameras [9]. Thirdly, the traced objects in the surveillance videos are much fewer than the sport video. So the occlusion problems between targets is more serious. All those make it more difficult to capture moving objects in the broadcast sports video, less accurate follow-up. Therefore, existing methods for detecting and tracking motional objects can't easily make desirable results [10-11].

Aiming at these problems, puts forward the method of detecting and tracking objects of robustness movement, to obtain the player trajectory information broadcast sports video. First, Support Vector Classification (SVC) combined with court segmentation. To detect the regional of player in sports video, initialize the target tracker. Secondly, Support Vector Regression (SVR) and Sequential Monte Carlo (SMC) framework are combining, the traditional Particle Filter (PF) algorithm is improved. The traditional particle filter method is robust tracking for visual objectsin the small particle sets.

\section{Player Detection based on Court Segmentation}

Color information is a low-level feature used in the paper for separation of the stadium. Currently color spaces which are used the most often are RGB, HSL, YUV, CIE L*a*b and so on. RGB is the most common, of which three components are red, green and blue. Digital images/videos are collected and saved in the form of RGB color space. The problem is the color space can't reflect well subjective characteristics of human's visual perception. All those color spaces, HSL is a type of subjective color model, presenting better the change of man's visual sensation. In this space, $\mathrm{H}$ refers to hue, for gifferent colors; $\mathrm{S}$ means saturation, for the shade of colors; L is luminanee for color intensity. Those three components, component $\mathrm{L}$ is more susceptible to outdoor illumination. $\mathrm{H}$ and $\mathrm{S}$ are decided by light absorption and reflection features of raw materials which make up physical objects. The method for converting RGB and HSL color space is expressed in Figure1. When the color information in the court is described, component H of HSL color space can indicate color types of the court; L may suggest visualeatures of different court colors under different illuminative conditions 12-13]. Thus, to create a model of court colors, it's necessary to use the two components of HSL color space [14].

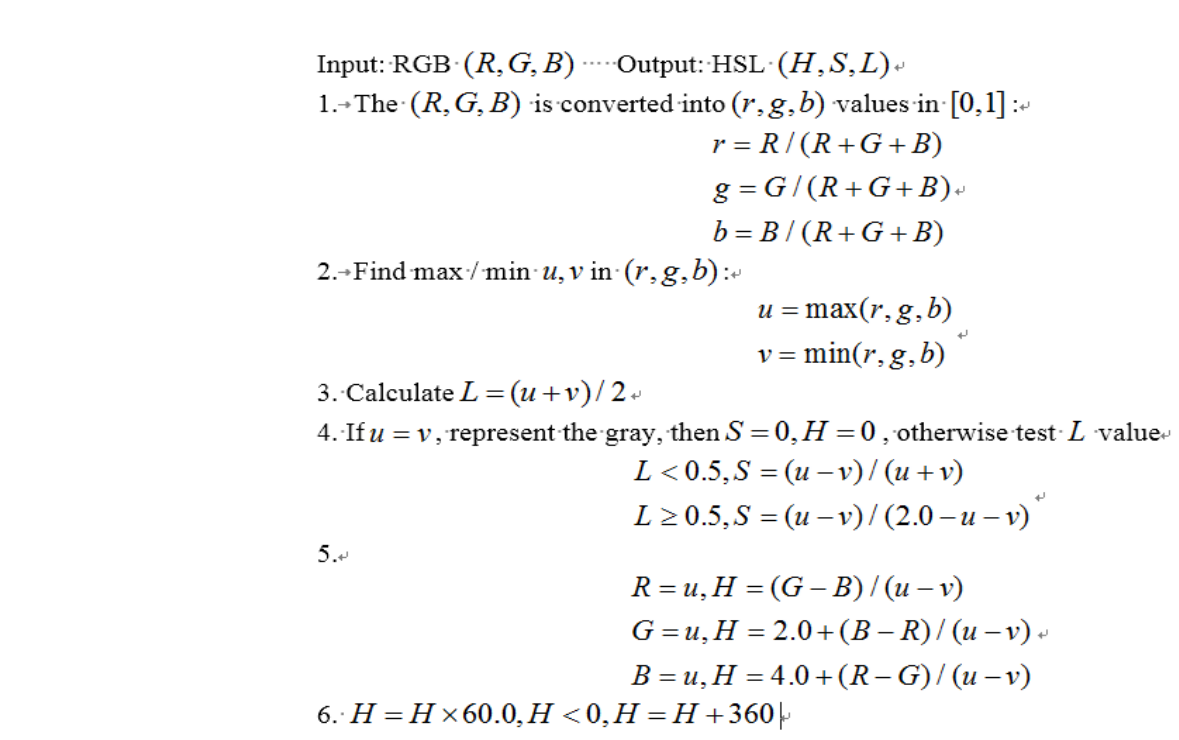

Figure 1. Conversion from RGB Color Space to HSL Color Space 


\section{Player Track Based on SVR and Particle Filtering}

\subsection{Introduction of Particle Filtering}

In the visual tracking system, the information of any possible location of traced objects can be modeling to the system status space based on Bayesian theory. Then according to the measurement of confidence level of each sampling point in the status space, we can have the posterior distribution of the location mentioned above. Due to existence of many uncertain factors like movements of traced objects, change of backgrounds and complexity and diversification of imaging conditions, the confident degree of location sampling points is of non-linear or non-Gaussian distribution in the system status space. In recent years, particle filtering theory has been successfully applied in the visual tracking field [15]. It has merits because it can not only make multiple hypotheses based on probability idea and also can deal well with non-linear or non-Gaussian distribution problems in the state space Particle filtering technique achieves iterative Bayesian filtering through non-parametric Monte Carlo simulations. It is suitable for any non-linear system which cân usse staté space model and traditional Kalman filtering method can't represent it, the precision approximate to the optimal estimation. The essential idea of that theory is try ing to utilize all known information to build the posterior distribution of system state varrables, ie., foreseeing the prior distribution of state with system model and then modifying observed values which are latest used to finally get the posterior distribution.

Particle filter algorithm flow shown in Figure 2, Particle predicts and updates through continuous iteration, so as to obtain the discrete estimation of posterior distribution. First, using the $p\left(x_{t} \mid x_{t-1}\right)$ dynamics model of tracked objectsto predict the original particles, get particles the current time $t$. Then, to obtain Observations of particles value, by the confidence function $p\left(z_{t} \mid x_{t}\right)$ of the particles to be updated. Then, to update the particles resampling to overcome the degeneracy problem.

Input: videof sequence $I(t)$, the initial state of the target object $x_{0}$

Output; the sequence state of tanget object $x_{1: T}$

1 - Initi lization, fron the initial distribution $p\left(x_{0}\right)$ generates particles $\left\{x_{0}^{i}\right\}_{i+1}^{N}, t=1$

2. $\rightarrow$ Particle prediction: from the distribution $x_{t}^{i} \sim p\left(x_{t} \mid x_{t-1}^{i}\right)$ sampling obtains prediction particles.

3.-Partice update: 'To measure Observation value $z_{t}$ of particle, 'using the confidence function $p\left(z_{t} \mid x_{t}^{i}\right)$ to assess the weights. $\tilde{w}_{i}^{t}=w_{t-1}^{i} \cdot p\left(z_{t} \mid x_{t}^{i}\right)$; And normalized. $8^{\circ}$ $w_{t}^{i}=\tilde{w}_{i}^{t} / \sum_{q=1}^{N} \tilde{w}_{t}^{q}, i=1, \ldots N$

4. $\rightarrow x_{t}=E\left(\left\{\left(x_{t}^{i}, w_{t}^{i}\right)\right\}_{i=1}^{N}\right)$

5. $\rightarrow$ Resampling: The use of a sampling strategy to get the new particle set $\left(x_{t}^{k}\right)_{k=1}^{N}$ from $\left(x_{t}^{i}\right)_{i=1}^{N}$ sampling

6. $\rightarrow$ IF $\cdot t<T$ then $t=t+1$, repeat step 2 , otherwise " output $x_{1: T}$ 'state sequence

Figure 2. Algorithm of Particle Filter 


\subsection{Problems with Traditional Particle Filtering Method}

The basic concept of particle filtering has roots in sequential Monte Carlo theory, using a set of state space sampling particles with weights to express the posterior distribution in a discrete manner. For the posterior distribution $p\left(x_{t} \mid z_{1: t}\right)$ at any time point $t$, we can use particle set at the point to approximate discretely as:

$$
p\left(x_{t} \mid z_{1: t}\right) \approx \sum_{i=1}^{N} w_{t}^{i} \delta\left(x_{t}-x_{t}^{i}\right)
$$

Which, $\delta(\cdot)$ is Dirac delta function. From formula1, we can learn that it's possible to improve the approximation degree of discrete expression to real distribution in two means. One is to increase the number of sampling particles. As Monte Carlo features suggest [16-17], when sampling data grow to infinitely many, the algorithm can approximate infinitely the real distribution of posterior possibility, so as to obtain the estimated results of infiniely close best solution. But in actual applications, overwhelming particles will reduce greatly the performance of particle filtering system. Meanwhile, it's unavoldable to have the problem of particle degeneracy in the filtering, to be specific, after iteration for some time, one particle will have the weight close to 1 , while others' weights are all very small. That means tremendous calculation will contribute very little to the final restlts. To overcome it, we put forward resampling method. The technique bases on the posterior distribution at the current moment to remove particles with small weights to give chances to those with bigger weights for evolution and survival. Resampling has the negative effect of SampleImpoverishment. Particles with bigger weights are repeatedly selected to catse many overlaps in the sampling results, destroying the variety of particles. Even though the use of small particles can help improve running efficiency of particle filtering, thaditional particle filtering can't make correct discrete expression of posterior distribution. Particle degeneration and deficit will become more serious.

\subsection{Improved Particle Filtering Algorithm based on Support Vector Regression}

To enhance the performance of particle filtering in the case of small particle sets, the evaluation of parficre weight is key point to deal with. Owing to weak robustness of confidence function, noises are generated in the posterior distribution. The kernel idea of the improved Support Vector RegressionParticle Filter algorithm based on support vectorRegression is to make use of such regression to get the formula of posterior distribution through online estimation, smoothing the distribution of noises and eliminating them. Then through re-assessment of particle weights, more accurate and real posterior distribution is ultimately acquired. As shown in Figure3.

\section{Moving Object Detection and Tracking Framework Based on SVM and Particle Filtering}

The detection and track of moving objects are to define the location of targets in images. But there're differences between them. Tracking uses not only objects' apparent characteristic information and also dynamic information to make it matched in the time axis. Tracking can't determine the initial position of objects. It needs to work with the aid of detection and tracking modules. Detection uses only objects' apparent characteristics to extract their locations, providing initial information to tracking. Tracking is helpful to improve detection effect and efficiency. For the purpose of prior sections, in this section, we'll integrate detection and tracking modules into a system in terms of applicability. Besides, in theoretical 
terms, the detection and tracking framework of common moving objects is shown in Figure4, which is based on SVM and particle filtering method. Statistics learning idea and random tracking theory are merged together. SVM theory has two applications: classification and regression. In the framework, the classifying method based on SVM theory is utilized to detect motional objects, providing initial targets to tracking devices. With Support vector Classification regression introduced to sequential Monte Carlo framework, the improved particle filtering tracking strategy is developed. The application of Support vector Classification regression enables better running efficiency and effect of the traditional filtering methods.

Input: video sequence $I(t)$, the initial state of the target object $x_{0}$

Output: the sequence state of target object $x_{1: T}$

$1 . \rightarrow$ Initialization: from the initial distribution $p\left(x_{0}\right)$ generates particles $\left(x_{0}^{i}\right)_{i=1}^{N}, t=1$

2. $\rightarrow$ Particle prediction: from the distribution $x_{t}^{i} \sim p\left(x_{t} \mid x_{t-1}^{i}\right)$ sampling obtains predic ion particles.

$3 \rightarrow$ Particle update: To measure Observation value $z_{t}$ of particle, "using the confidence function $p\left(z_{t} \mid x_{t}^{i}\right)$ to assess the weights. $\tilde{w}_{i}^{t}=w \cdot p\left(z_{t} \mid x_{t}^{i}\right)$, and normalized $w_{t}^{i}=\tilde{w}_{i}^{t} / \sum_{q=1}^{N} \tilde{w}_{t}^{q}, i=1, \ldots N$

4. $\rightarrow$ Evaluation of particle weight based ' on sapport vector regression:- Using ' support vector regression method based on particle set $\left\{\left(x_{t}^{i}, w_{t}^{3}\right)\right\}$ The regression equation $f_{t}(x)$ of online estimation of the posterior dist 1bution. To evaluate the particle weights using $f_{t}(x)$ to get updafe particle $\{(x, w)\}_{i=1}$

$5 . \rightarrow x_{t}=E\left(\left\{\left(x_{t}^{i}, w_{t}^{i}\right)\right\}_{i=1}^{N}\right)$

6. $\rightarrow$ Resampling: The use of a sampling strategy to get the new particle set $\left(x_{t}^{k}\right)_{k=1}^{N}$ from $\left(x_{t}^{i}\right)_{i=1}^{N}$ sampling

$7 . \rightarrow \mathrm{IF} t<T$ then $t=\Omega$, , repeat step 2 , otherwise output $x_{1: T}$ state sequence

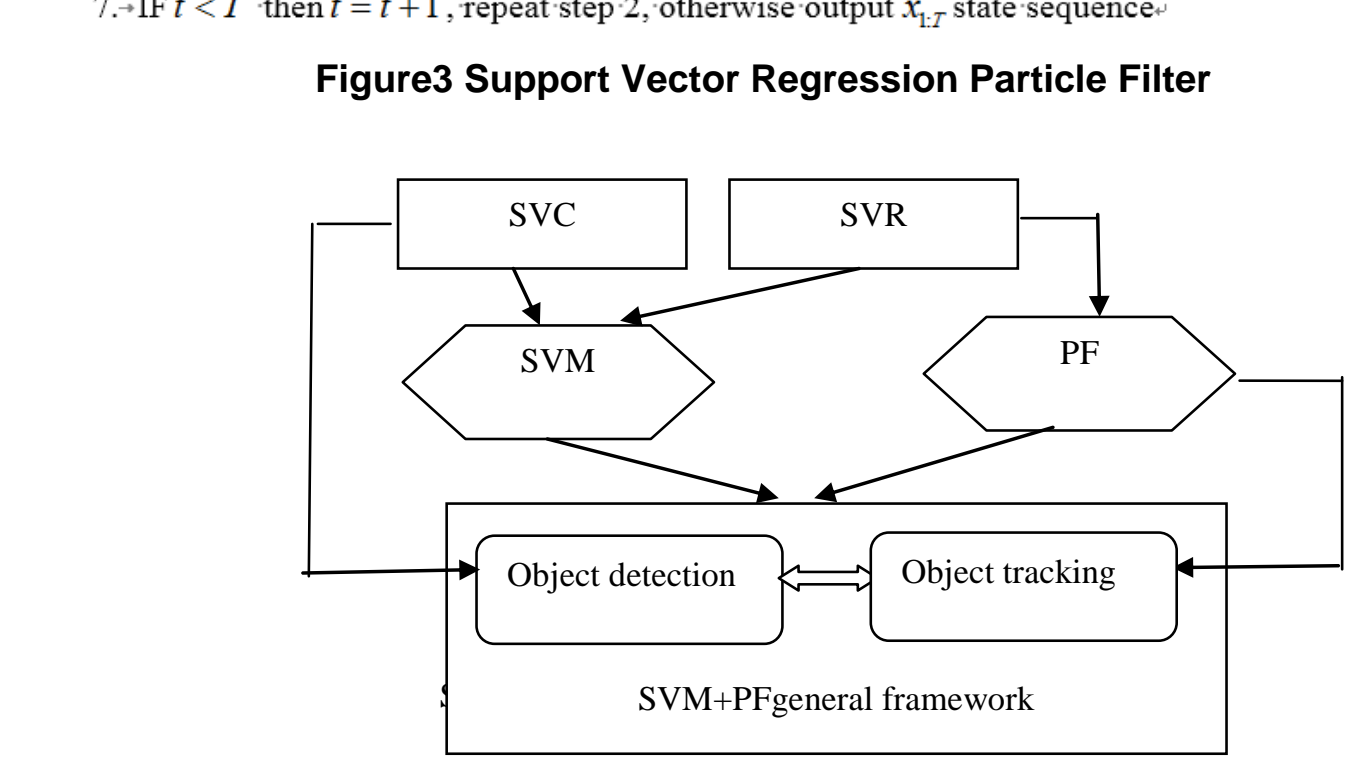

Figure4 Unified Framework for MovingObject Detection and Tracking based on SVMand PF 


\section{Experiment Design and Results}

In this section, we test the performance of proposed algorithm for the real video data from three aspects: Player detection result. Comparison results Support vector regression particle filter with the traditional particle filter. Player tracking result.

To validate the algorithm generality, we used five types of sports event videos to make the test, which are football, hockey, badminton, tennis and basketball videos. Every competition video is directly transcribed from broadcast TV programs, stored in MPEG-2 compact format, with video frame size $352 \times 288$. Table 1 lists out all experimental data, including frames of visual sequence and SUMof Player (SOP). The point is how to define SUM of Player. For given video sequence $V, S O P$ can be defined like:

$$
\operatorname{SOP}(V)=\sum_{i=1}^{N} m_{i}
$$

Which, $N$ is a sequence of $V$ contains frames, $m_{i}$ is the number of the $i$ player sequence in $V . i=1, \ldots, N$.

Table1 Experimental Data of Player Detection and Tracking

\begin{tabular}{|c|c|c|}
\hline Video sequence & Frames & SO P \\
\hline soccer-chunk-1 & 466 & 1232 \\
\hline soccer-chunk-2 & 300 & 795 \\
\hline soccer-chunk-3 & $\mathbf{3 3 6}$ & 1241 \\
\hline soccer-chunk-4 & 242 & 763 \\
\hline hockey-chunk-1 & 491 & 2904 \\
\hline hockey-chunk-2 & 384 & 1233 \\
\hline hockey-chunk-3 & 476 & 1640 \\
\hline badminton-chunk-1 & 199 & 398 \\
\hline badminton-chunk-2 & 237 & 474 \\
\hline badminton-chunk-3 & 354 & 708 \\
\hline tennis-chunk-1 & 693 & 1386 \\
\hline tennis-chunk-2 & 226 & 452 \\
\hline tennis-chunk-3 & 475 & 950 \\
\hline tennis-chunk-4 & 431 & 862 \\
\hline basketball-chunk-1 & 165 & 594 \\
\hline basketball-chunk-2 & 138 & 287 \\
\hline all & 5613 & 15919 \\
\hline
\end{tabular}

\subsection{Testing Results of Player Inspection}

In order to appraise the performance of the proposed method for detecting player, we experimented over all sixteen video sequences. In the experiment, we collected samples at the even interval of 10 for every video frame to train the Gaussian mixture model of court colors. When constructing Support vector Classificationin the detected player area, to avoid overlearning problem, we took three-times cross validation strategy, i.e. every time two thirds of training samples are chosen as training set, with the remaining as testing set. The operation repeats three times. The training results which can achieve the best classifying effect is selected as the final classifier. Table I shows the experimental results of the method. Here "success rate" $(A)$ can be defined as: 


$$
A=\frac{n_{\text {detected }}}{n_{\text {total }}} \times 100 \%
$$

Which, $n_{\text {det ected }}$ means player area which is detected; $n_{\text {total }}$ refers to all player area included in the video sequence, which is now $S O P$ value. As observed from Table2, performance of the proposed method is desirable. Leak detection happened for the following reasons:

5.1.1 Too small area of player led to misjudge the after-selected areas to noisy areas in the background when such areas are extracted after division of the court, e.g. in the hockey competition, player area is much smaller than in other contests; the area of far-away player in the tennis video is relatively smaller too, who're staying away from cameras when the event is rebroadcast.

5.1.2Player areas are too close to tag lines and overlaying subtitles in the video frame or rebroadcasting TV station caption, which are converged to the neighboring areas when morphological operation of regional extraction is performed. That is usually seen from football video sequence and distance player in tennis video;

5.1.3 Serious overlapping between players makes the area of two members form one, which caused the missing detection. It is commonly foundin the football orbasketball match when player are fighting harder to contact too closely with one another;

5.1.4Large shadows on the playground make player area too dark to check out some place. Shades are quite common. For that point, the un-detection is found in every video sequence.

Table2 Experimental Results of Player Detection

\begin{tabular}{|c|c|c|c|c|}
\hline Video sequence & Frames & SO P & $\begin{array}{c}\text { ctect players } \\
\text { region }\end{array}$ & $\begin{array}{c}\text { The success } \\
\text { rate(100\%) }\end{array}$ \\
\hline soccer-chunk-1 & 466 & 1232 & 1025 & 83.2 \\
\hline soccer-chunk-2 & 300 & 795 & 639 & 80.4 \\
\hline soccer-chunk-3 & 336 & 1241 & 946 & 76.2 \\
\hline soccer-chunk-4 & 242 & 763 & 634 & 83.1 \\
\hline hockey-chunk-1 & 491 & 2904 & 2288 & 78.8 \\
\hline hockey-chunk-2 & 384 & 1233 & 979 & 79.4 \\
\hline hockey-chunk-3 & 476 & 1640 & 1331 & 81.2 \\
\hline badminton-chunk-1 & 199 & 398 & 379 & 95.2 \\
\hline badminton-chunk-2 & 237 & 474 & 460 & 97.0 \\
\hline badminton-chunk-3 & 354 & 708 & 669 & 94.5 \\
\hline tennis-chunk-1 & 693 & 1386 & 1253 & 90.4 \\
\hline tennis-chunk-2 & 226 & 452 & 431 & 95.4 \\
\hline tennis-chunk-3 & 475 & 950 & 934 & 98.3 \\
\hline tennis-chunk-4 & 431 & 862 & 837 & 97.1 \\
\hline basketball-chunk-1 & 165 & 594 & 416 & 70.0 \\
\hline basketball-chunk-2 & 138 & 287 & 230 & 80.1 \\
\hline all & 5613 & 15919 & 13451 & 84.5 \\
\hline
\end{tabular}

\subsection{Comparison Results Support Vector Regression Particle Filter with the Traditional Particle Filter}

In the aim of comparing the performance of the improved particle filtering method with the old, we experimented in soccer-chunk-4. The traditional methods used in the experiment are displayed in Figure2, where is also the new method based on Support vector regression in 
Figure 3. To assess objectively the tracking method, traced areas were manually marked to initialize trackers, protecting the method from being affected by other factors. Tracked areas were expressed in their exterior rectangle. For the two methods of tracking, state space is expressed by four-dimensional vectors as seen in the following:

$$
x=\left(x, y, v_{x}, v_{y}\right)^{T}
$$

Which, $(x, y)$ is the image coordinates of the upper left corner of the rectangular region tracking, $\left(v_{x}, v_{y}\right)$ represents movement speed of the image along rectangular region of $\mathrm{x}$-axis, $\mathrm{y}$-axis.Dynamic model of the tracked object is defined:

$$
x_{t}=A \cdot x_{t-1}+n_{t}
$$

Which, $n_{t}$ is compatible with $N(0.5)$ distribution of the random noise, based on motion speed of player the state transition matrix $A$ hypothesis is defined:

$$
A=\left\{\begin{array}{llll}
1 & 0 & 0.9 & 0 \\
0 & 1 & 0 & 0.9 \\
0 & 0 & 1 & 0 \\
0 & 0 & 0 & 1
\end{array}\right)
$$
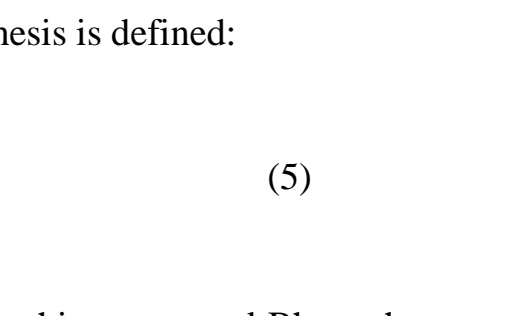

Tracking region is similarity based on HSV color histogram and Bhattacharyya, we define confidence function of the particle filter algorithin.

$$
p(z \mid x) \propto \exp \left(-\lambda \cdot \Delta^{2}\left[q^{*}, q(x)\right]\right)
$$

Which, $\lambda=20$ is a constant, $D^{2}\left[q^{*}, q(x)\right]$ is distance Based on Bhattacharyya similarity definition template histogram $q^{*}$ and the tracked Region Histogram $q(x)$.

$$
D^{2}\left[q^{*}, q(x)\right]=\left(1-\sum^{N} \sqrt{(i) \cdot q(x, i)}\right)^{1 / 2}
$$

$N$ is the number of histogram bin Two tracking algorithms are used 50 particles in the experiment.

In order to tes the tracking efficiency,we increase the particle size of the traditional particle filter method,in order to achieve tracking effect of support vector regression similar particle filter. When the 200 particles, two tracking method is similar.At this point, the traditional particle filtertracking time consumption for the whole video sequence is 1.8 times than the support vector regression particle filter.It is evident, although we added a step in the original particle filter algorithm, however, this step enables to increase the efficiency of the algorithm and improve the running time is much greater than the cost of their own.Therefore, the support vector regression particle filter to improve the operating efficiency of the tracking system.

\subsection{Experimental Results of Tracking Player}

We combined both detection and tracking methods to do complete test of tracking playing members. The detection and tracking tests were performed alternately. If the traced area is determined a non-player one by the player tracing classifier after consecutive $L=10$ frame, the track is deemed failure. We need to run again the detection algorithm. Testing results are seen in Table 3.

The algorithm performance is affected mainly by player occluding with one another. When the occlusion exists for a long time, such as player close-fitting struggling for balls in the 
football match, the shaded player position and the positional relation between forward and backward frames can't be recognized nor judged even by naked eyes. In that case, the method here considers such position or path as the information of the sheltered player. For player who are sheltered, the tracking device can't acquire the information of their right locations, which are deemed non-player areas after being identified by support vector classifier in lots of frames. Thus traced targets are lost. When shadows disappear, we can re-operate the method to acquire player area and initialize new trackers to track again.

\section{Conclusion}

This paper studies for the detection and tracking of player in broadcast sports video. First, proposed player detection algorithm based on support vector classification and Court Segmentation. The player candidate regionis obtained based on the course of segmentation.Use offline training a supervised classifier to identify candidate region,get accurate player area in the game scene.Based on the analysis of the problems existing in the traditional particle filter, and proposes an improved particle filter algorith $n$ based on support vector regression. This improvement not only increases the particle filter racking effect on small particles, but also improve the operation efficiency of the particle filter tracking system.Experimental results show that the method is proposed in this paper, the players of different sports broadcast video detection and tracking is very effective.

Table3 Experimental Results of Player Tracking
\begin{tabular}{|c|c|c|c|c|}
\hline Video sequence & Frames & SoP & $\begin{array}{c}\text { detect players } \\
\text { region }\end{array}$ & $\begin{array}{c}\text { The success rate } \\
(100 \%)\end{array}$ \\
\hline soccer-chunk-1 & 466 & 1232 & 1088 & 88.3 \\
\hline soccer-chunk-2 & 300 & 75 & 693 & 87.2 \\
\hline soccer-chunk-3 & 336 & 1241 & 994 & 80.1 \\
\hline soccer-chunk-4 & 242 & 763 & 680 & 89.1 \\
\hline hockey-chunk-1 & 491 & 2904 & 2517 & 86.7 \\
\hline hockey-chunk-2 & 384 & 1233 & 1045 & 84.8 \\
\hline hockey-chunk-3 & 476 & 1640 & 1466 & 89.4 \\
\hline badminton-chunk-1 & 199 & 398 & 383 & 96.2 \\
\hline badminton-chunk-2 & 237 & 474 & 474 & 100.0 \\
\hline badminton-chunk-3 & 354 & 708 & 708 & 100.0 \\
\hline tennis-chunk-1 & 693 & 1386 & 1365 & 98.5 \\
\hline tennis-chunk-2 & 226 & 452 & 452 & 100.0 \\
\hline tennis-churk-3 & 475 & 950 & 950 & 100.0 \\
\hline tennis-chunk-4 & 431 & 862 & 862 & 100.0 \\
\hline basketball-chunk-1 & 165 & 594 & 457 & 76.9 \\
\hline basketball-chunk-2 & 138 & 287 & 242 & 84.3 \\
\hline and & 5613 & 15919 & 14289 & 89.8 \\
\hline
\end{tabular}




\section{References}

[1] D. Liang, Q. Huang, Y. Liu, et al.,"Video2cartoon: A System for Converting Broadcast Soccer Video Into3d Cartoon Animation”, IEEE Trans. on Consumer Electronics, vol. 53, no. 3, (2007), pp. 1138-1146.

[2] J. Assfalg, M. Bertini, C. Colombo, et al.,"Semantic Annotation of Soccer Video:Automatic Highlights Identification. Computer Vision and Image Understanding”, vol. 92, no 2-3, (2003), pp. 285-305.

[3] H. Miyamori and S. Iisaku,"Video Annotation for Content-based Retrieval Using Hu-man Behavior Analysis and Domain Knowledge", IEEE International Conference on Automatic Face and Gesture Recognition. Grenoble: IEEE, (2000), pp. 320-325.

[4] G. Sudhir, J. Lee and A. Jain,"Automatic Classification of Tennis Video for High-level Content-based Retrieval", IEEE International Workshop on Content-Based Access of Image and Video Databases. Bombay: IEEE, (1998), pp. 81-90.

[5] Y. Sun andK. Lee, "Video compression coding effects on motion detection", Information technology, vol.02, (2013), pp. 126-129.

[6] J. Zhao, X. Sun and L. Wu, "Dynamic target substation particle filter tracking technology based on the inside", Journal of Beijing Jiaotong University, vol.4, (2013), pp. 153-157.

[7] F. Xu, X. Zheng and L. Tao, "The tracking method for soccer video HOG and particle fitter based on the ball”, Electronic technology, vol. 9, (2013), pp. 36-40.

[8] H. Xiang, "The application of virtual reality technology in college basketball broadcasts", China broadcast TV, vol. 12, (2013), pp. 42-43.

[9] Q. Wang andL. Xia, "tan on it, Prediction of the player with the ball in basketball video China behavior", Journal of image and graphics, vol.4, (2012), pp. 560-567.

[10] P. Qu, S. Qu, T. Kang andY. Zhao,"Analysis of sports video intelligence based on low-level visual information", Journal of sports adult education, vol. 3, (2012), pp. 49-51.

[11] X. Zhang, H. Liu andY. Sun,"An adaptive multiple features fusion player tracking algorithm”, Computer Engineering, vol. 17, (2012), pp. 214-217.

[12] S. Jiang, Q. Ye, W. Gao, et al.,"A New Method to Segment Playfield and its Applications in Match Analysis in Sports Video", ACM International Conference on Multimedia New York: ACM, (2004), pp. 292-295.

[13] Q. Ye, W. Gao and W. Zeng, "Color Image Segnentation Using Density-based Clustering", IEEE International Conference on Acoustics, Speech, and Signal Processing, Hong Kong: IEEE, vol. 2, (2003), pp. 401-404.

[14] P. Perez, C. Hue, J. Vermaak, et al.,"Color Based Probabilistic Tracking",European Conference on Computer Vision, Copenhagen: Springer, (2002), pp. 661-675.

[15] M. Isard and A. Blake, "Condensation-conditional Density Propagation for VisualTracking", International Journal of Computer Vision vol. 29, no. 1, (1998), pp. 5-28.

[16] A. Doucet, "On Sequentral Simulation-based Methods for Bayesian Filter", Tech.Rep. CUED/FINFENG/TR310, University of Cambridge, (1998).

[17] R. Merwe, A. Doucet, N. Freitas, et al." "The Unscented Particle Filter", Tech.Rep. CUED/FINFENG/TR380, University of Cambridge, (2000).

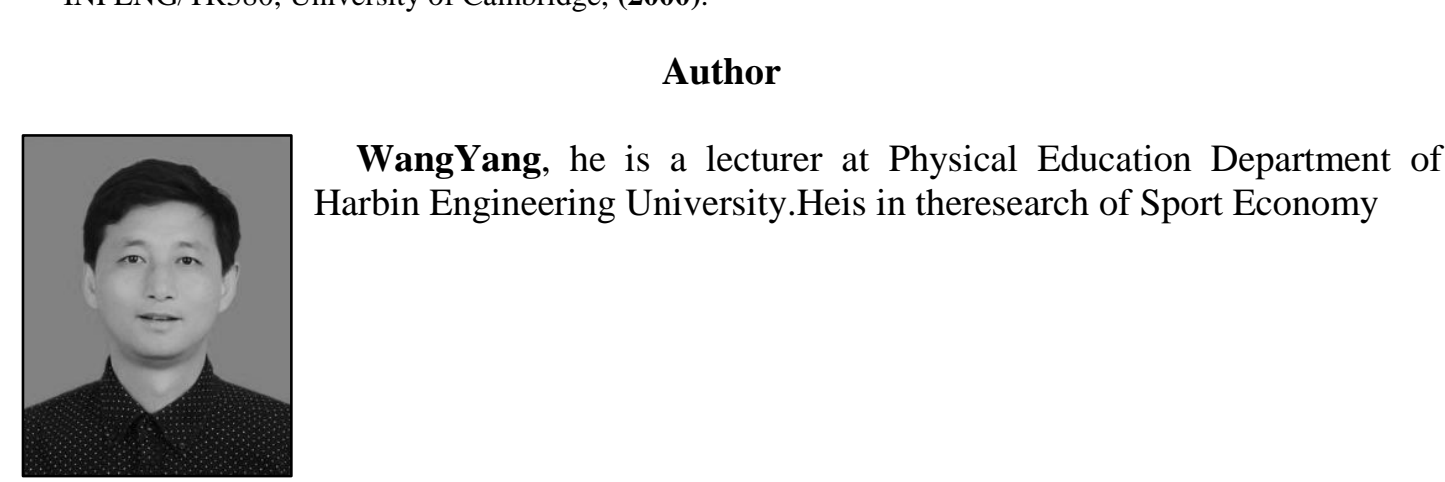

